At a symposium of Section $H$ in 1948 at Brighton, the effect of technique on social institutions was considered, but the conclusions suffered from lack of data on the time factor. More recently McCarthy and McArthur made a careful study of the time occupied in the food quest by two groups of Australian aborigines, from which significant conclusions could be drawn. Further, Manning Nash has recently published a study of pottery-making in a Mexican village in which he showed that the overall factor determining the output of pots was the incidence of fiestas. He also drew general conclusions on the impediment to pottery-making caused by the presence in a family of young children and of the advantage held by families where there were enough women to introduce something akin to production line mothods. The value of these generalizations would have been enhanced if the time involved in the various processes of manufacture had been obtained.

The method of timing operations will be difficult in large or complicated societies, but by careful sampling, combined with orthodox methods of anthropological research, valuable information should be obtained. It is most important to obtain information on primitive techniques before it is too late.

\section{LIPID CO-FACTORS IN BIOCHEMISTRY}

N his presidential address to Section I (Physiology and Biochemistry), Prof. R. A. Morton considers "Lipid Co-factors in Biochemistry".

Many enzymes have prosthetic groups, which may be detachable coenzymes. These sometimes contain a water-soluble vitamin which is the locus of the substrate-coenzyme interaction.

It is reasonable to postulate that fat-soluble vitamins may also act as co-factors in enzymatic processes. The modes of action of lipid co-factors present difficult problems and progress has been relatively slow.

The concept of a vitamin is now somewhat blurred. Thus vitamin $\mathrm{A}$ has carotonoid precursors which in the animal organism yield vitamin A alcohol. This is followed by ester formation and liver storage, and although the vitamin is mobilized as alcohol it may function as a derived compound. Similarly, cholesterol is synthesized by the animal and dehydrogenated in the gut wall to yield provitamin $D_{3}$, which yields some cholecalciferol (vitamin $\mathrm{D}_{3}$ ) under the action of ultra-violet light. The status of vitamin $D$ as a dietary essential is thus weakened although its efficacy under test conditions is not in doubt. Vitamin E also has lost simplicity; the term tocopherol now refers to several natural substances varying in 'activity'. The distribution of tocopherols in plant and animal tissues does little more than emphasize an antioxidant role.

Vitamin $K_{1}$, with its phytyl side-chain, is found only in plant products and in fact has become somewhat elusive. The numerous vitamins $K_{2}$ are 2-methyl-1,4-naphthoquinones with a polyisoprenoid side-chain at position 3 . Vitamin $K_{2(20)}$ has a $\mathrm{C}_{20} \mathrm{H}_{33}$ side-chain and is peculiar to animals. Vitamins $K_{2(30)}, K_{2(35)}, K_{2(40)}, K_{2(45)}$ are all found in microorganisms. Vitamin K-antagonists point to an enzymatic role and the lack of sharp specificity of chemical structure in relation to 'activity' raises difficult problems. $\alpha$-Lipoic acid leads to a useful linking of a fat-soluble co-factor with enzymatic processes catalysed by thiamine pyrophosphate.

The blurring of the vitamin concept has been followed by the discovery of new endogenous lipid co-factors which are readily synthesized but which deserve to be called essential metabolites. Thus plastoquinone (a 2,3-dimethyl-1,4-benzoquinone with a $\mathrm{C}_{45} \mathrm{H}_{73}$ side-chain at position 6) occurs in the chloroplasts of leaves and is perhaps more significant in photosynthesis than vitamin $K_{1}$. The alcohol solanesol $\left(\mathrm{C}_{45} \mathrm{H}_{73} \mathrm{OH}\right)$ occurs in plants and a cyclic isomeride of plastoquinone, solanachromeno, is obtained from tobaceo. The ubiquinones are 5methyl-2,3-dimethoxy-1,4-naphthoquinones with a polyisopronoid side-chain $\left(\mathrm{C}_{5} \mathrm{H}_{8}\right)_{n}-\mathrm{H}$, where $n$ is $6-10$.

The compound ubichromenol $(50)$ is isomeric with ubiquinone (50) and bears the same relationship to the latter as solanachromene does to solanesol.

With regard to lipid co-factor functions real progress has been made, but baffling difficulties persist. For example, retinene ${ }_{1}$ (retinal or vitamin A aldehyde) is the key compound in vision, with special emphasis on one cis-isomer which combines with an amino group in a specific protein before a visual pigment can be formed. Vitamin A acid (retinoic acid) can fulfil some of the systemic functions of vitamin $\mathbf{A}$ alcohol (retinol) but it cannot sustain vision, reproduction or some aspects of bone growth. It may be that vitamin A alcohol is the precursor of a more powerful but very labile catalyst.

The evidence on vitamin D suggests that in rats it tends to prevent rickets, when the causative low phosphate intake is marginal.

Vitamin $\mathrm{E}$ occurs in animal tissues and its distribution in cell components does not exclude a major catalytic role, despite complicating evidence that synthetic anti-oxidants permit reproduction over three generations on a vitamin F-free diet. The multiplicity of syndromes in different species and the response of some, but not all, to catalytic amounts of selenite or organically bound selenium complicates an already difficult situation.

The possibility that $\alpha$-tocopherol, vitamin $\mathrm{K}$ and ubiquinone function in electron transport and oxidative phosphorylation has been much canvassed, and will be discussed by Dr. F. A. Holton and Dr. E. R. Redfearn. Plastoquinone is a key substance in photosynthesis and in photophosphorylation. Vitamins $\mathrm{K}$ seem to function in plants and in many bacteria. Some micro-organisms produce ubiquinones while others produce vitamins $\mathrm{K}$, and a few produce both. Tocopherols on oxidation yield quinones, and it seems that quinones may act as phosphate carriers via chromans or chromenes.

Knowledge of the modes of action of lipid cofactors is in transition; most of the pieces of the puzzle are possibly to hand and bits of the picture are probably correctly assembled, but tho major synthesis has still to be made. The discovery of new quinones has transformed several topics and new techniques have radically altered the approach via enzymology and biosynthesis.

\section{IDENTIFICATION AND USE OF TALENT}

$T$ ALENT in the sense of general mental efficiency, operational and latent, actual and potential, is identified and assessed by psychologists by means of tests. This forms the theme of Mr. Denis MeMahon's presidential address to Section J (Psychology). These have certain advantages, but some sciontific difficulties can arise which can lead to circular arguments. From test evidence, which is backed by other evidence, it is clear that even before the 11-plus stage many able children have 